\title{
Article \\ PAPR Impact over the PER of Vehicle-to-Vehicle Communications with Fading Channels
}

\author{
Italo Alexander Carreño, Frank Andrés Eras 0000-0002-7844-5670, Thomas Borja, Diego Javier \\ Reinoso* 0000-0003-0854-1250, Luis Felipe Urquiza 0000-0002-6405-2067, Martha Cecilia \\ Paredes 0000-0001-5789-4568 \\ Departamento de Electrónica, Telecomunicaciones y Redes de Información, Escuela Politécnica Nacional \\ * Correspondence: diego.reinoso@epn.edu.ec
}

\begin{abstract}
Peak-to-Average Power Ratio (PAPR) is one of the main problems in wireless communications using Orthogonal Frequency Division Multiplexing (OFDM). Its behavior is random and can produce problems for the hardware implementation, directly influencing the Packet Error Rate (PER). In this article, the PER is obtained for channels with Rayleigh and Rician fading. In the simulation, a High Power Amplifier (HPA) is added to the transmitter and for PAPR reduction Simple Amplitude Predistortion-Orthogonal Pilot Sequences (OPS-SAP) technique is used.
\end{abstract}

Keywords: PAPR; HPA; OPS-SAP; PER; fading models

\section{Introduction}

Orthogonal Frequency Division Multiplexing (OFDM) is a digital communication scheme that transmits using multiple orthogonal carriers [1]. It is an attractive technology for wireless communications because it offers considerable high spectral efficiency, multipath delay spread tolerance, immunity to the frequency selective fading channels and power efficiency [2].

One of the main drawbacks of the OFDM system is the high Peak-to-Average Power Ratio (PAPR) of transmitted OFDM signals. The PAPR in OFDM transmission can be defined as the ratio of peak power with respect to average power. A high PAPR requires that the power amplifiers work in a very large linear region, otherwise, the saturation of the devices occurs, causing out-of-band radiation that affects adjacent bands and within the band that attenuates and displaces the signal, increasing the Bit Error Rate (BER) [3].

The simplest way of modeling a wireless communication channel is using the Additive White Gaussian Noise (AWGN). However, for a more accurate model it is necessary to take into account the fading, which is the variation of the signal in time and frequency, caused mainly by the multipath, lost by trajectory and by shadows due to obstacles. The main two models for the fading are Rayleigh and Rician that are used for NLOS (Non-Line-of-Sight) and LOS (Line-of-Sight), respectively.

\section{Theoretical Review}

\subsection{IEEE 802.11p Comunication System}

The OFDM physical layer model will follow the training and reception process described in the IEEE 802.11 standard [4] using an AWGN, Rayleigh, and Rician channel. With a bandwidth of 10 $\mathrm{MHz}$, the eight possible combinations of coding rate and modulation scheme available in the IEEE 802.11 standard [4] that allow to obtain different speeds for data transmission are implemented, these combinations are shown in Table 1. 


\begin{tabular}{|c|c|c|c|}
\hline $\begin{array}{c}\text { Modulation } \\
\text { Scheme }\end{array}$ & $\begin{array}{c}\text { Bits Per } \\
\text { Modulated } \\
\text { Symbol }(m)\end{array}$ & $\begin{array}{c}\text { Coding } \\
\text { Rate }(r)\end{array}$ & $\begin{array}{c}\text { Data } \\
\text { rate } v_{i} \\
{[\mathbf{M b p s}]}\end{array}$ \\
\hline BPSK & 1 & $1 / 2$ & 3 \\
\hline BPSK & 1 & $3 / 4$ & 4.5 \\
\hline QPSK & 2 & $1 / 2$ & 6 \\
\hline QPSK & 2 & $3 / 4$ & 9 \\
\hline 16QAM & 4 & $1 / 2$ & 12 \\
\hline 16QAM & 4 & $3 / 4$ & 18 \\
\hline 64QAM & 6 & $2 / 3$ & 24 \\
\hline 64QAM & 6 & $3 / 4$ & 27 \\
\hline
\end{tabular}

Table 1. Configurations allowed for IEEE 802.11p. [4]

\subsection{PAPR Problem}

The major obstacle in the transmitter side of any OFDM system, occurs when the signal has a very high peak instantaneous power with respect to its average power, due to the possibility that the subcarriers can be aggregated with identical phase in a certain time, thus the time-domain signal achieves a very important contribution of power [5]. The signal peaks lead to certain hardware components such as Digital-Analog Converters and Analog Amplifiers to have a behavior below their maximum performance.

\subsection{Channel Models}

\subsubsection{AWGN Model}

It is the simplest channel model in a wireless communications environment. The mathematical expression in received signals that passed through the AWGN channel is: $r(t)=s(t)+n(t)$ where $s(t)$ is the transmitted signal and $n(t)$ is noise. It is the basic communication channel model and it is used as a standard channel model since the transmitted signal gets disturbed by a simple additive white Gaussian noise process [6].

\subsubsection{Rayleigh Fading Model}

Rayleigh fading is mainly caused by multipath reception. It is a statistical model for the effect of a propagation environment on a radio signal. It is a reasonable model for the propagation of troposphere and ionosphere signals, as well as the effect of highly urbanized urban environments on radio signals. Rayleigh fading is most applicable when there is no line of sight (NLOS) between the transmitter and the receiver [6].

\subsubsection{Rician Fading Model}

Rician fading can be defined as a Rayleigh fading model, but the difference lies in the dominant component. In the Rician fading channel model, there is a strong dominant component present that is a non-fade signal commonly known as the line of sight component. This fading occurs when one of the paths has a stronger line of sight than others [7].

\subsection{High Power Amplifier}

The aim of The High Power Amplifier (HPA) is to increase the amplitude of the signal by a factor $\mathrm{K}$. The main characteristic of the HPA is that they do not have a linear behavior. The most efficient operating point for an HPA is at the saturation level. However, high peaks encountered in OFDM signals can drive the nonlinear HPA into saturation [5]. In practical systems, the HPA is a device limited in power and very sensitive to the variation of the amplitude of the signal. The type of HPA most commonly used is Solid State Power Amplifier (SSPA) implemented with the Rapp Model. 


\subsection{PAPR reduction techniques}

Currently, several techniques have been proposed to reduce PAPR in OFDM systems. They can be classified into techniques that introduce signal distortion, and those that do not introduce signal distortion. Each of the techniques has its advantages as disadvantages and the analysis of each of the different techniques is not covered in this work.

One of the main techniques to reduce PAPR is the Simple Amplitude Predistortion together with Orthogonal Pilot Sequences (OPS-SAP), with which it is possible to move certain constellation points of the OFDM symbol to counteract the PAPR[8].

\subsubsection{OPS-SAP technique}

This technique for reducing PAPR is based on a two-step algorithm, where the OPS is implemented in the first step and then SAP is added as the second step. In the first stage the sequence of pilots that offers the lowest PAPR, of the whole set of available pilots, is inserted. In the second step, the extension of certain symbols in the frequency domain is performed, the symbols to be extended are chosen by means of a metric, which measures the contribution of the frequency symbols that have large power peaks in the time domain [5].

\section{Matlab implementation}

\subsection{Rayleigh and Rician block diagrams}

The packing, scrambler, convolutional coder, interleaver, modulator, OFDM symbol, Cyclic prefix aggregation, cyclic prefix extraction, OFDM symbol mapping, demodulator, deinterleaver, convolutional decoder, and unpacking generator blocks were obtained from the scripts related to the thesis document [9]. They create, compose and decompose OFDM symbols and packets.

The Up frequency block receives OFDM packets as input. A carrier frequency vector with the same size as the OFDM packet is generated and it is applied to the packet to achieve the up frequency. The opposite process is performed at reception (Down frequency block), the carrier frequency vector is applied to the packet to change it to baseband.

The PAPR reduction technique block receives OFDM symbols as input, and its PAPR is obtained. If this value is over $6 \mathrm{~dB}$, OPS is applied. The amplifier block is carried out by using the Rapp model. The block uses IBO and s parameters set at the start of simulation.

The application of the fading channel block is done by applying MATLAB's own functions: comm.Rayleigh and comm.Rician. It receives OFDM packets as input. These functions also generate the channel frequency response used later by the equalization block. The AWGN channel block is also applied by a MATLAB's own function. It uses the values given by the SNR vector and the OFDM packet as inputs.

The equalization block receives OFDM symbols at reception. The frequency response from the symbol is obtained and then divided by the conjugate of the frequency response of the fading channel.

\section{Simulation Results}

Figures 1, 2 and 3 present PER vs SNR curves for amplified passband OFDM frames when they cross an AWGN, Rayleigh and Rician channel, respectively. An equalization process was carried out for Rayleigh and Rician channel cases. Dashed line curves represent OFDM frames without the application of the PAPR reduction technique while solid line curves represent OFDM frames with the application of the PAPR reduction technique.

These figures show a minimal difference in the PER when the OPS-SAP technique is used for low data rates (until $18 \mathrm{Mbps}$ ). For $24 \mathrm{Mbps}$ and $27 \mathrm{Mbps}$ data rates a considerable difference is observed. In this case the PAPR reduction technique is able to improve the PER significantly. 
PER performance is similar in the cases of AWGN and Rician. However, the Rayleigh case presents a decrease in performance of $5 \mathrm{~dB}$ on average compared with the AWGN case. This is caused due to Rayleigh fading since there is no line of sight in this type of fading.

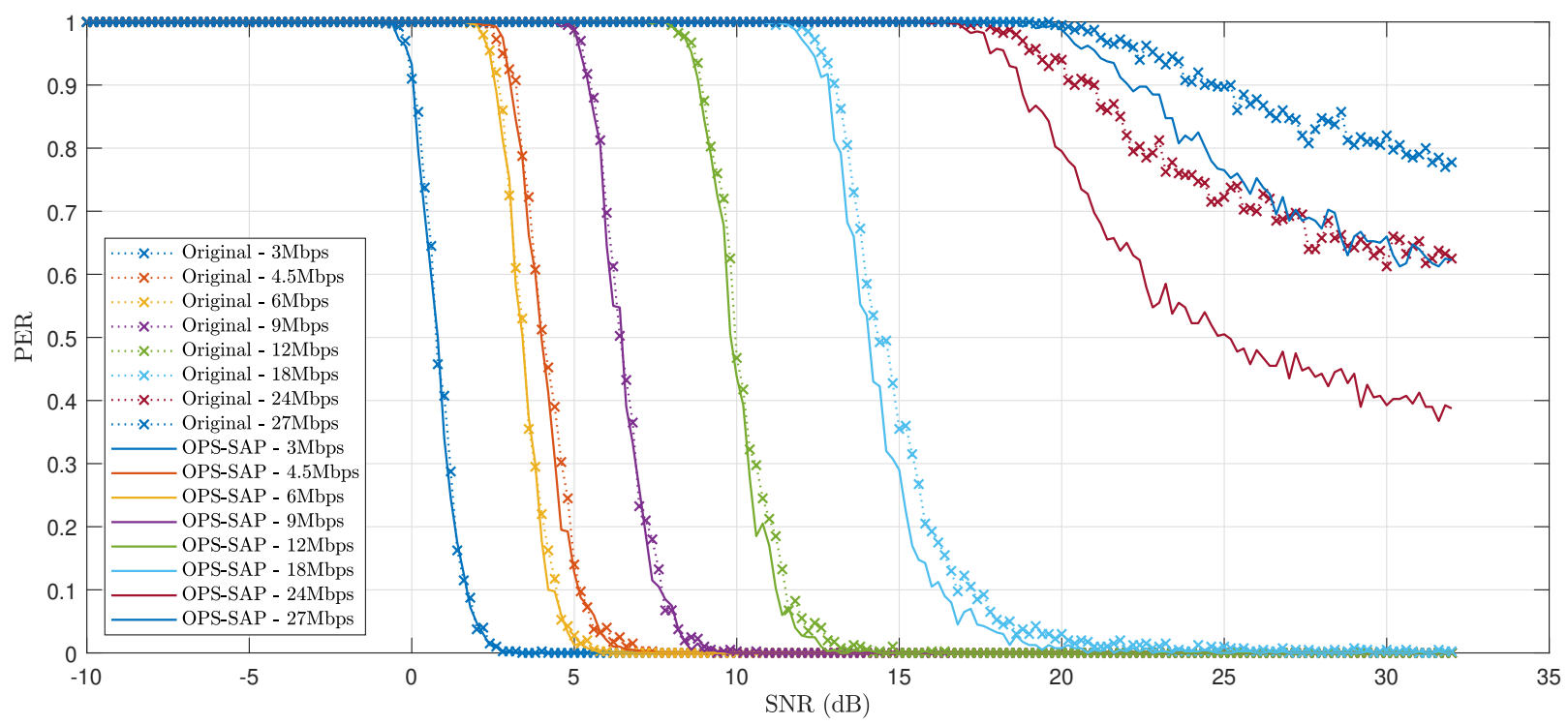

Figure 1. PER vs SNR of amplified passband signal (5.9 GHz) with AWGN channel, SSPA, IBO=6 dB).

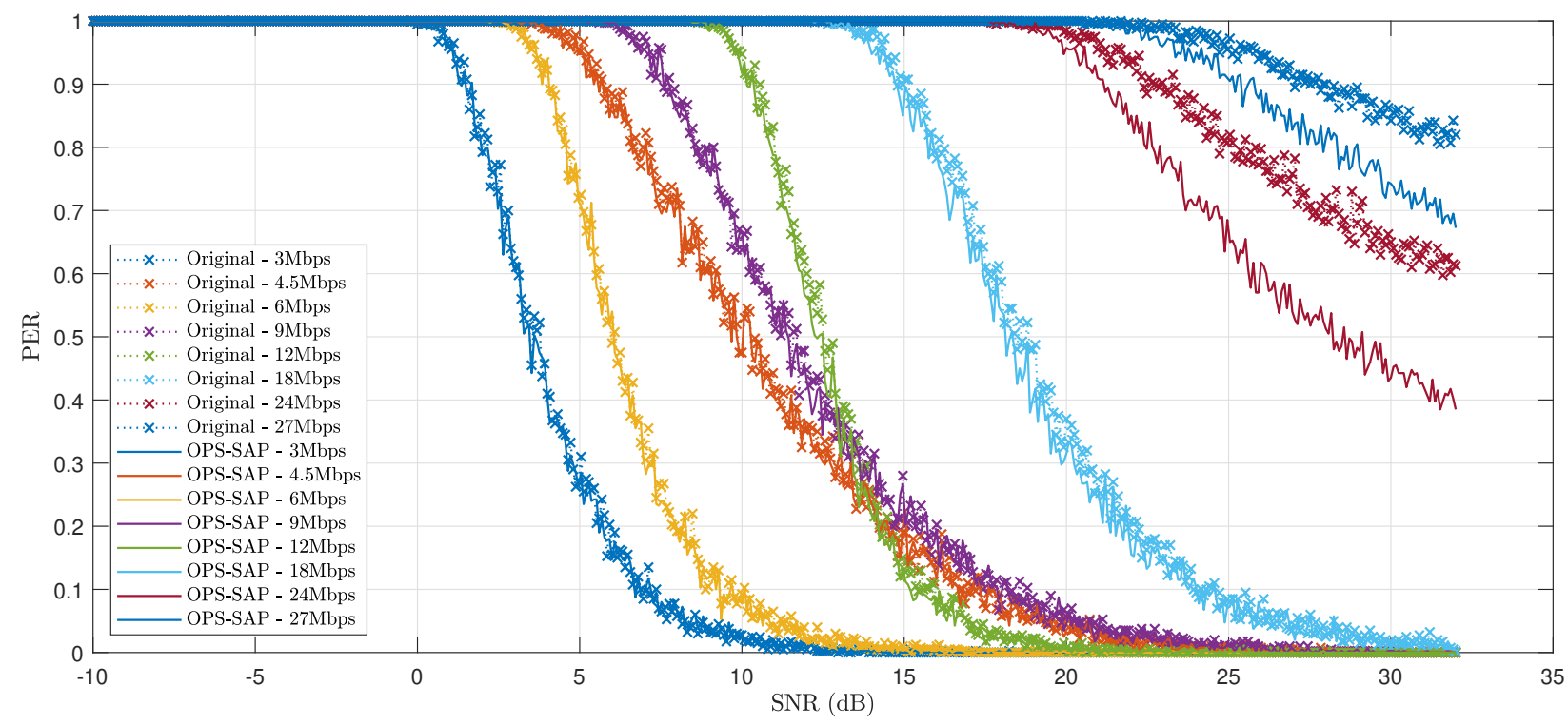

Figure 2. PER vs SNR of amplified passband signal $(5.9 \mathrm{GHz})$ with Rayleigh fading channel, SSPA, $\mathrm{IBO}=6 \mathrm{~dB}$. 


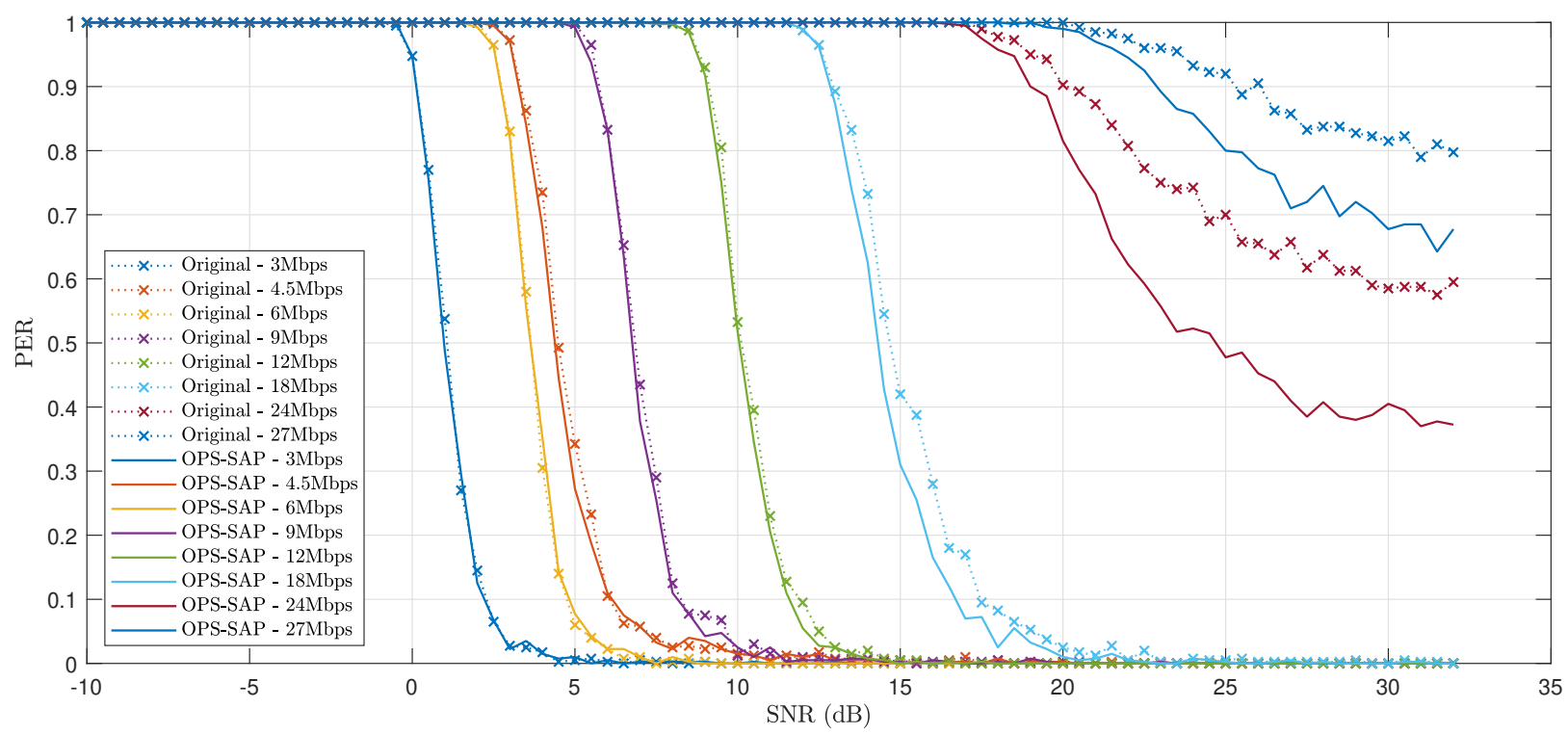

Figure 3. PER vs SNR of amplified passband signal $(5.9 \mathrm{GHz})$ with Rician fading channel, SSPA, IBO=6 dB.

Figures 4, 5, and 6 present PER vs SNR curves for amplified baseband OFDM frames when they cross an AWGN, Rayleigh and Rician channel respectively. Similarly to the previous figures, dashed line curves represent OFDM frames without the application of the PAPR reduction technique whilst solid line curves represent OFDM frames with the application of the PAPR reduction technique. These figure do not show a considerable difference when comparing frames with and without technique which leads to the conclusion that PAPR reduction technique does not affect the PER performance significantly. Same as passband frames, PER performance is similar in the cases of AWGN and Rician. However, the Rayleigh case presents a decrease in performance of $8 \mathrm{~dB}$ on average compared with the AWGN case. This is caused due to Rayleigh fading since there is no line of sight in this type of fading.

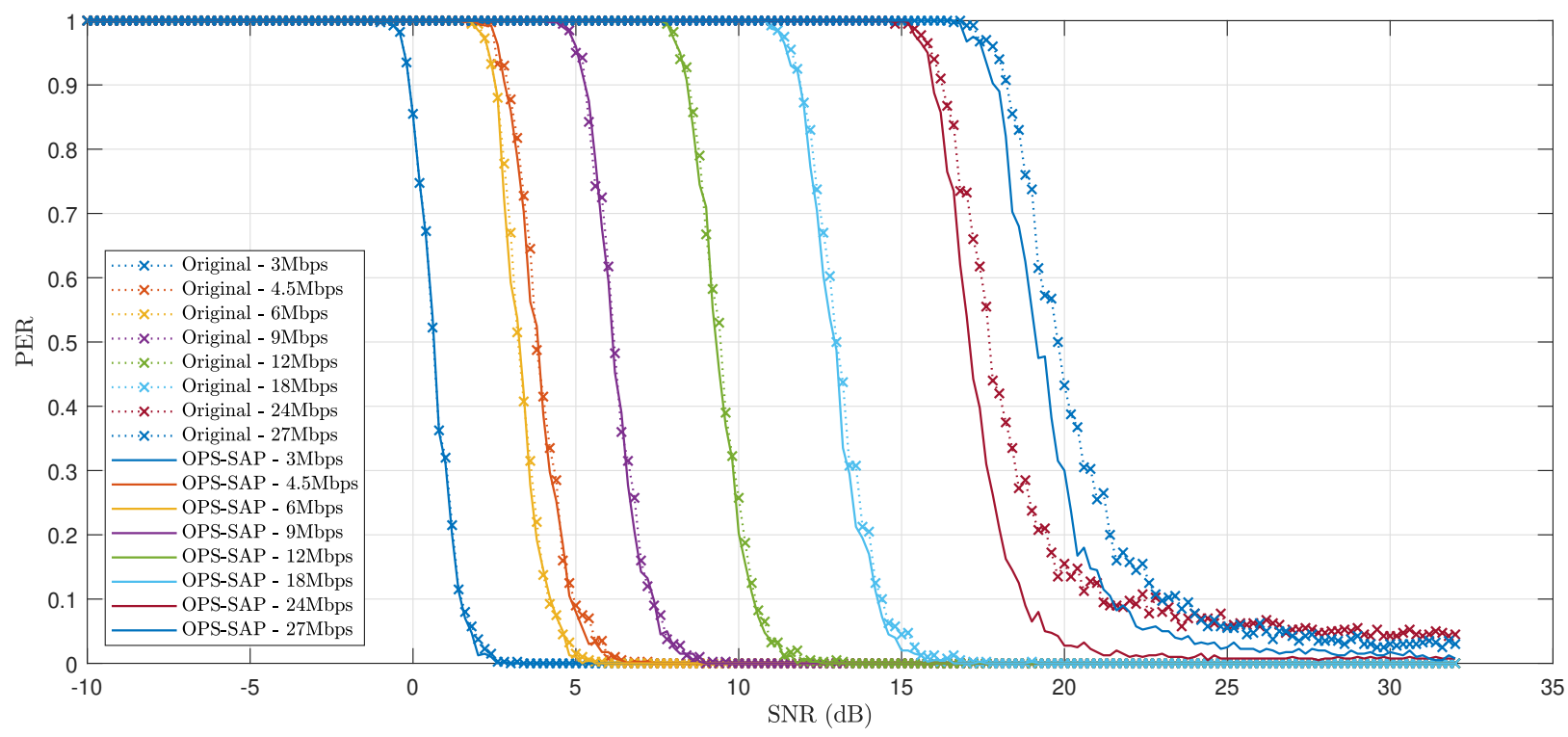

Figure 4. PER vs SNR of amplified baseband signal with AWGN channel, SSPA, IBO=6 dB 


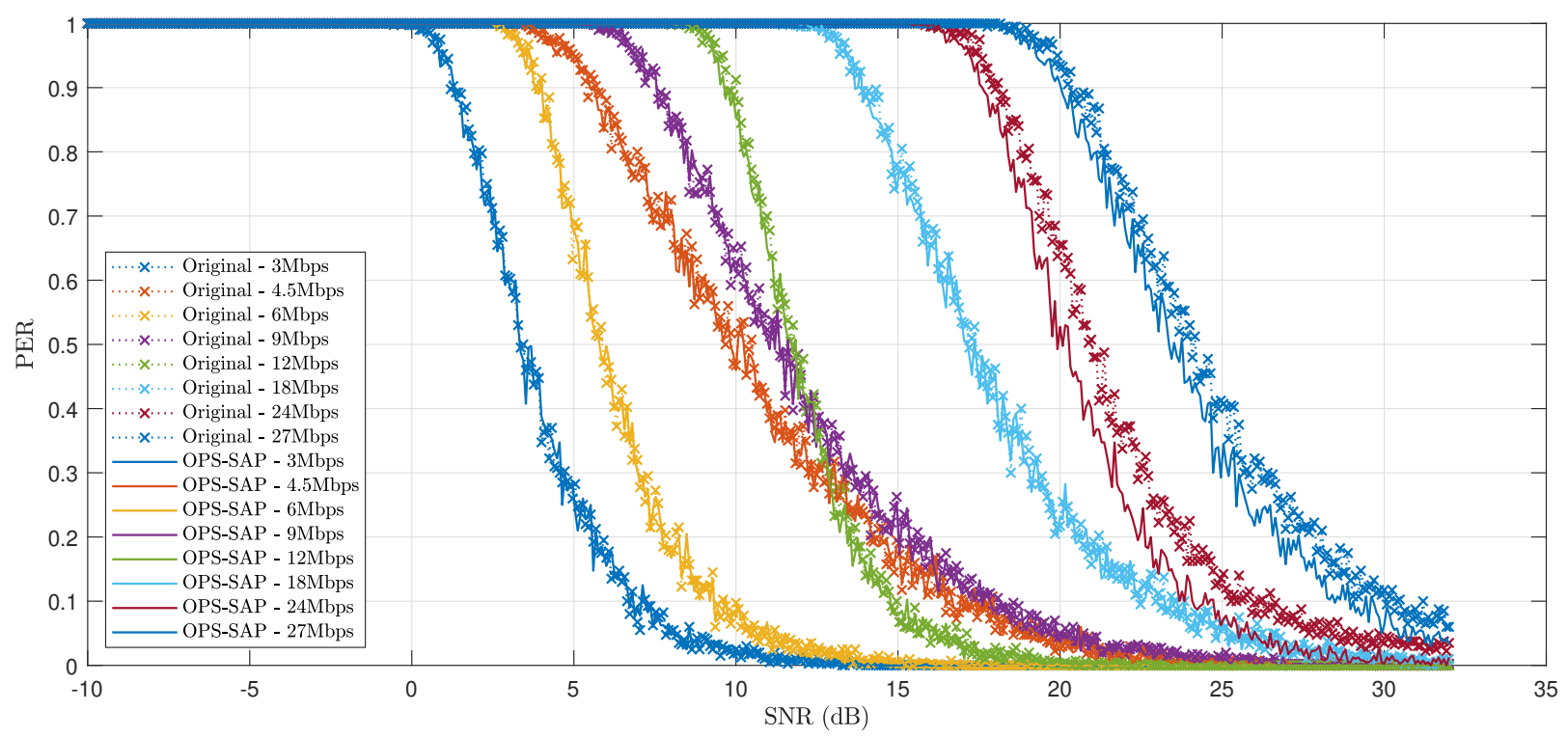

Figure 5. PER vs SNR of amplified baseband signal with Rayleigh fading channel, SSPA, IBO=6 dB

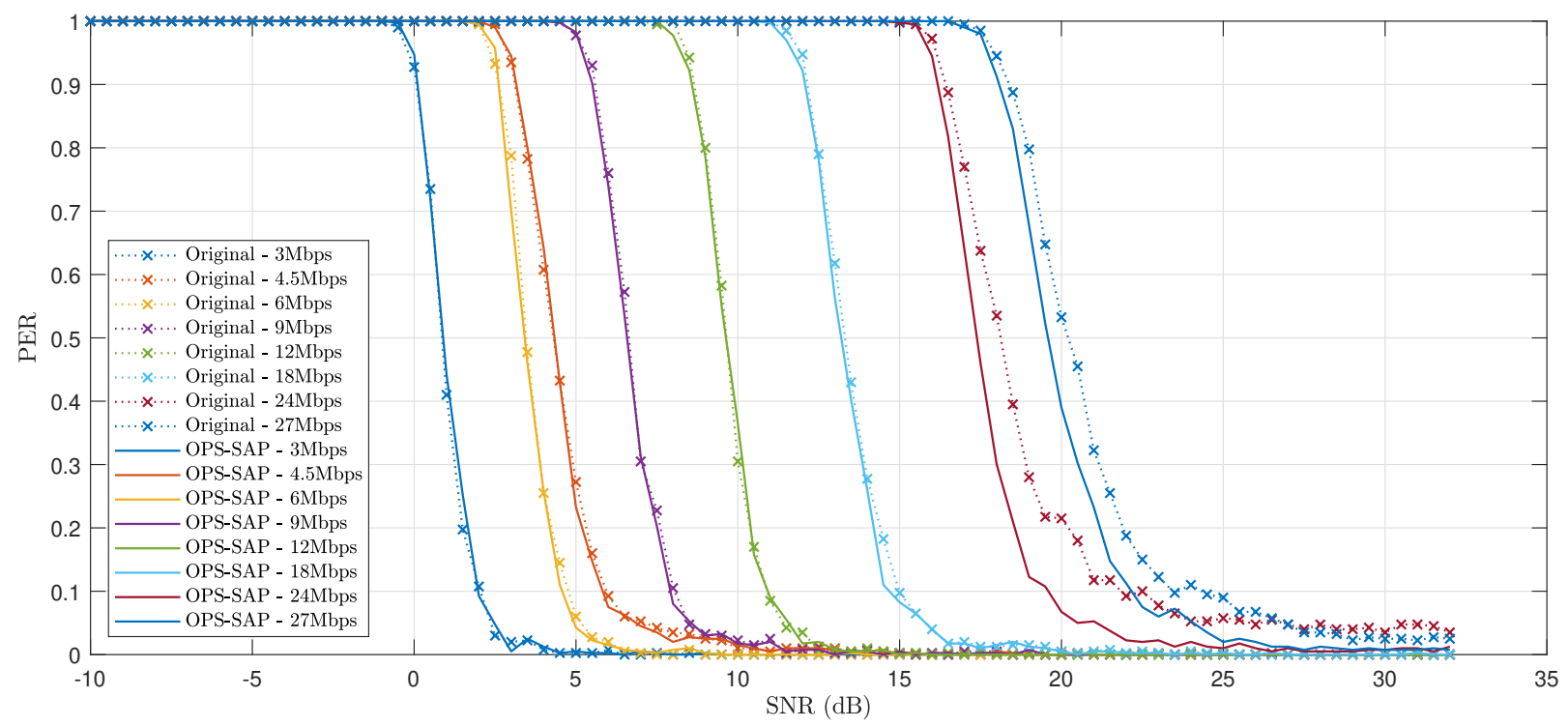

Figure 6. PER vs SNR of amplified baseband signal with Rician fading channel , SSPA, IBO=6 dB

\section{Conclusions}

- For high data rates (24 Mbps and $27 \mathrm{Mbps}$ ) the use of the OPS-SAP technique for the reduction of the PAPR is of great importance due to the improvement that the curves show since they are displaced to the left.

- The channel with the best PER is the AWGN followed by the Rician channel and finally the Rayleigh channel. There are very few differences between the AWGN channel and Rician. On the other hand, the Rayleigh channel shifts the curves to the right in several dBs, taking the AWGN channel as a reference, in addition to the aforementioned, the Rayleigh channel is the only one that presents nonlinearities in its curve, in other words it varies a lot on a small scale but maintaining the characteristic form. 
- Even though the AWGN channel obtains the best PER, the Rayleigh and Rician channel models are widely used because they are closer to the reality.

1. Lakshmi, M.V.; Kanmani, B. PAPR reduction through lossy coding. 2017 International Conference on Algorithms, Methodology, Models and Applications in Emerging Technologies (ICAMMAET), 2017, pp. 1-4. doi:10.1109/ICAMMAET.2017.8186704.

2. Pandey, P.; Tripathi, R. Performance Analysis of Peak-to-Average Power Ratio (PAPR) Reduction Techniques in an OFDM System. 2012 Third International Conference on Computer and Communication Technology, 2012, pp. 245-249. doi:10.1109/ICCCT.2012.57.

3. Paredes, M.C.P. Algoritmos para la reducción de los picos de potencia en los sistemas OFDM. PhD thesis, Universidad Carlos III de Madrid, Escuela Politécnica Superior, Departamento Teoría de la señal y comunicaciones, 2014.

4. IEEE Standard for Information technology-Telecommunications and information exchange between systems Local and metropolitan area networks-Specific requirements Part 11: Wireless LAN Medium Access Control (MAC) and Physical Layer (PHY) Specifications. IEEE Std 802.11-2012 (Revision of IEEE Std 802.11-2007) 2012, pp. 1-2793. doi:10.1109/IEEESTD.2012.6178212.

5. Paredes, M.C.P.; Navarrete, D.M.; Placencia, D.F.P. Analysis of quantization metrics for PAPR evaluation in OFDM systems. 2016 8th IEEE Latin-American Conference on Communications (LATINCOM), 2016, pp. 1-6. doi:10.1109/LATINCOM.2016.7811602.

6. Babu, A.; Rao, K. "Evaluation of BER for AWGN, Rayleigh and Rician Fading Channels under Various Modulation Schemes". International Journal of Computer Applications 2011, 26, 23-28. doi:10.5120/3132-4317.

7. Vashist, S.; Singh, R. BER and PAPR Analysis of OFDM and SC-FDMA for Different Fading Channels 2016. pp. 2278-9359.

8. Paredes, C.; García, M. Performance of OPS-SAP technique for PAPR reduction in IEEE 802.11p scenarios. Ad Hoc Networks 2016, 52. doi:10.1016/j.adhoc.2016.07.010.

9. Cabezas, X.A.F. Análisis cualitativo y cuantitativo de modelos para el cálculo del PER (Packet Error Rate) para comunicaciones IEEE 802.11p. PhD thesis, Escuela Politécnica Nacional, 2019. 